\title{
AIRFOIL IN A SONIC SHEAR FLOW JET: \\ A MIXED BOUNDARY VALUE PROBLEM FOR THE GENERALIZED TRICOMI EQUATION*
}

BY

\author{
C. C. CHANG AND T. S. LUNDGREN \\ University of Minnesota
}

\begin{abstract}
In this paper, small perturbations of a non-uniform two-dimensional flow of a compressible inviscid fluid are considered. It is shown that for a particular class of main stream Mach number distributions, which are characterized by a sonic line along the $x$-axis, the linearized shear flow equation may be transformed into the generalized Tricomi equation. The mixed boundary value problem which results from considering perturbations generated by a two-dimensional camber surface is formulated and solved by utilizing the Wiener-Hopf technique.

1. Introduction. In recent years, Lighthill [1] Chang and Werner [2] and others have treated two-dimensional linearized compressible shear flow problems. In this paper a parallel but non-uniform stream of compressible fluid is perturbed by a thin two-dimensional airfoil which is located along a sonic line. The main stream Mach number distribution, $M(y)$, resembling that approached by a sharp edged sonic jet under the influence of viscosity, is assumed symmetrical about the sonic line at $y=0$.

Let $p(x, y)$ be the perturbation pressure, $P$ be the constant freestream pressure, and $\alpha(x, y)$ be the slope of the streamlines in the disturbed flow. Also let $M_{0}=M(0) . \alpha$ and $p$, if small enough, can be determined from a function $\phi(x, y)$ satisfying the linear eqution [3]

$$
\left(1-M^{2}\right) \frac{\partial^{2} \phi}{\partial x^{2}}+\frac{\partial^{2} \phi}{\partial y^{2}}-\frac{2}{M} \frac{d M}{d y} \frac{\partial \phi}{\partial y}=0 .
$$

$\phi$ is related to $p(x, y)$ and $\alpha(x, y)$ by

$$
\begin{gathered}
\frac{\partial \phi}{\partial x}=\frac{p}{(\gamma / 2) P M_{0}^{2}}=C_{p}, \\
\frac{\partial \phi}{\partial y}=-2 \frac{M^{2}}{M_{0}^{2}} \alpha .
\end{gathered}
$$

Here $C_{p}$ is the local pressure coefficient and $\gamma$ the ratio of specific heats. It is generally accepted that (1.1) is a valid linearization of the equations of fluid motion provided that $d M / d y$ is large enough at points where $M=1$. This condition is not satisfied for the type of Mach number profile considered in this paper. It appears reasonable, however, that (1.1) is valid if higher derivatives of $M$ are large enough at sonic points.

Let the airfoil have unit chord length and be a camber surface so that the slopes of the upper and lower surfaces both equal $\alpha_{0}(x)$. The condition that the flow be tangent to the airfoil can be obtained from (1.3) as

$$
\frac{\partial \phi(x, 0)}{\partial y}=-2 \alpha_{0}(x), \quad 0<x<1 .
$$

*Received September 3, 1958. 
For subsonic flows, the disturbance must die out at infinity. This condition can be accomplished by imposing that $\phi$ approaches zero at infinity. The problem can be somewhat simplified by specifying boundary data along the whole $x$-axis instead of only on the airfoil. It is shown in Appendix I that, as in incompressible potential flow $\phi$ has a jump discontinuity which is equal to the lift coefficient, $C_{l}$. This jump can be taken conveniently to be along the positive $x$-axis behind the airfoil. In addition, because of the symmetry of the Mach number profile and the anti-symmetry of the airfoil, the transformation $y \rightarrow-y, \phi \rightarrow-\phi$ leaves Eqs. (1.1) and (1.4) unchanged. This implies that $\phi$ is an odd function of $y$. This fact and the jump condition require

$$
\begin{aligned}
\phi\left(x, 0_{+}\right) & =0 & & x<0 \\
& =-C_{\imath} / 2 & & x>1 .
\end{aligned}
$$

Equations (1.4) and (1.5) give mixed boundary data along the entire $x$-axis.

In general, solutions of (1.1) are difficult to obtain with any arbitrarily given $M(y)$. However, as will be shown in Part II, for a particular choice of $M(y)$, Eq. (1.1) can be transformed to the form

$$
Y^{n} \frac{\partial^{2} \phi}{\partial x^{2}}+\frac{\partial^{2} \phi}{\partial Y^{2}}=0, \quad Y \geqq 0,
$$

where $n$ is a non-negative constant and $Y$ is a new independent variable

$$
Y=b \int_{0}^{y} M^{2}(y) d y \quad b=\text { constant. }
$$

Eq. (1.6) is commonly known as the generalized Tricomi equation. This equation has been widely studied as shown in the bibliography to [4]. However, no solution is known for the above mixed boundary conditions.

The present treatment will be divided into two parts. In Part I, the mathematical solution to this problem will be derived. In Part II, the application to an airfoil in a sonic shear flow jet and the details of the corresponding Mach number profile will be considered.

\section{Part I. Solution of the Generalized Tricomi Equation with Mixed Boundary Conditions}

2. Statement of problems and procedure for solution. The problem is to solve the generalized Tricomi equation

$$
Y^{n} \frac{\partial^{2} \phi}{\partial x^{2}}+\frac{\partial^{2} \phi}{\partial Y^{2}}=0, \quad Y \geqq 0, \quad n \geqq 0
$$

with the mixed boundary conditions

$$
\begin{aligned}
& \phi(x, 0)=0, \quad x<0 ; \\
& \phi(x, 0)=-\frac{C}{2}, \quad x>1 ; \\
& \frac{\partial \phi(x, 0)}{\partial Y}=\theta(x), \quad 0<x<1 ; \\
& \phi(x, \infty)=0 .
\end{aligned}
$$


Here $C$ is a constant and $\theta(x)$ is bounded and integrable. If $\phi(x, 0)$ were given along the whole $x$-axis, the problem would be a Dirichlet problem which could be solved easily by use of classical techniques such as the Fourier integral or two-sided Laplace transforms. By applying Wiener-Hopf techniques $[5,6]$ to the present problem, the missing Dirichlet data, $\phi(x, 0)$ on the interval $(0,1)$, can be found. This is carried out in two steps. In Sec. 3 a related auxiliary problem is first solved, namely, the generalized Tricomi equation with $\partial \phi(x, 0) / \partial Y$ specified on the positive $x$-axis, and $\phi(x, 0)=0$ on the negative $x$-axis ${ }^{1}$. This is done with the aid of two-sided Laplace transforms and the Wiener-Hopf technique. In the main problem $\partial \phi(x, 0) / \partial Y$ is given on $(0,1)$ and $\phi(x, 0)$ is specified on $(1, \infty)$, consequently, the auxiliary problem gives an integral equation for the unknown quantities $\phi(x, 0)$ on $(0,1)$ and $\partial \phi(x, 0) / \partial Y$ on $(1, \infty)$. In Sec. 4 this integral equation is solved by using Mellin transforms with the Wiener-Hopf technique.

3. Solution for $\phi(x, 0)$ on $(0, \infty)$ when $\partial \phi(x, 0) / \partial Y$ is specified on $(0, \infty)$ and $\phi(x, 0)=$ 0 on $(-\infty, 0)$. If $\phi(x, Y)$ is a function which satisfies

$$
Y^{n} \frac{\partial^{1} \phi}{\partial x^{2}}+\frac{\partial^{2} \phi}{\partial Y^{2}}=0, \quad Y \geqq 0, \quad n \geqq 0
$$

and the boundary conditions

$$
\begin{aligned}
& \phi(x, 0)=0, \quad x<0 ; \\
& \frac{\partial \phi(x, 0)}{\partial Y}=\theta_{1}(x), \quad x>0 ; \\
& \phi(x, \infty)=0,
\end{aligned}
$$

it will be shown that on the positive $x$-axis, the solution is

$$
\phi(x, 0)=\frac{a^{-1}}{\mathrm{I}^{2}(1 / n+2)} \int_{0}^{x}(x-\xi)^{(1 / n+2)-1} d \xi \int_{\xi}^{\infty} \theta_{1}(\eta)(\eta-\xi)^{(1 / n+2)-1} d \eta,
$$

where

$$
a=-(1 / n+2)^{+n / n+2} \Gamma(n+1 / n+2) / \Gamma(1 / n+2) .
$$

First, let the boundary conditions (3.2) be restated as

$$
\begin{aligned}
& \phi(x, 0)=f_{1}(x) H(x), \\
& \frac{\partial \phi(x, 0)}{\partial Y}=\theta_{1}(x) H(x)+\theta_{2}(x) H(-x), \\
& \phi(x, \infty)=0,
\end{aligned}
$$

where $f_{1}(x)$ and $\theta_{2}(x)$ are unknown functions and $H(x)$ is the Heaviside step function, i.e.,

$$
\begin{aligned}
H(x) & =1, & & x>0 \\
& =0, & & x<0 .
\end{aligned}
$$

'This problem differs from that of Carrier [7] in that Carrier's boundary data are given on a line perpendicular to the parabolic (sonic) line. 
Secondly, let

$$
\Phi(s, Y)=\int_{-\infty}^{\infty} e^{-s x} \phi(x, Y) d x
$$

be the two-sided Laplace transform of $\phi(x, Y)$. Taking the Laplace transform of (1.1) and (3.4) gives

$$
\begin{aligned}
& Y^{n} s^{2} \Phi+\frac{\partial^{2} \Phi}{\partial Y^{2}}=0 \\
& \frac{\partial \Phi(s, 0)}{\partial Y}=\theta_{1}(s)_{+}+\theta_{2}(s)_{-}, \\
& \Phi(s, 0)=F_{1}(s)_{+}, \\
& \Phi(s, \infty)=0,
\end{aligned}
$$

where $\theta_{1}(s)_{+}, \theta_{2}(s)_{-}$and $F_{1}(s)_{+}$are defined by

$$
\begin{aligned}
& \Theta_{1}(s)_{+}=\int_{0}^{\infty} e^{-s x} \theta_{1}(x) d x \\
& \theta_{2}(s)_{-}=\int_{-\infty}^{0} e^{-s x} \theta_{2}(x) d x \\
& F_{1}(s)_{+}=\int_{0}^{\infty} e^{-s x} f_{1}(x) d x
\end{aligned}
$$

Some imposed properties of these functions and their consequences are given below:

(i) The given function $\theta_{1}(x)$ is assumed to approach zero like exp $(-\epsilon x)$ as $x \rightarrow \infty$; thus $\theta_{1}(s)_{+}$will be analytic for $\operatorname{Re}(s)>-\epsilon$ where $\epsilon>0$. Since $\theta_{1}(x)$ is a bounded function, $\theta_{1}(s)_{+}=0|s|^{-1}$ as $s \rightarrow \infty$.

(ii) $\Theta_{2}(s)_{-}$, an unknown function which is to be determined, must approach zero algebraically as $x \rightarrow-\infty$ so that $\theta_{2}(s)_{-}$will exist for $\operatorname{Re}(s)=0$ and be analytic for $\operatorname{Re}(s)<0$. The assumption $\theta_{2}(x)=0(-x)^{-p}, p<1$ as $x \rightarrow 0$ assures that $\theta_{2}(x)$ is integrable near the origin. This condition implies that $\theta_{2}(s)_{-}=0|s|^{-1+p}$ as $|s| \rightarrow \infty$.

(iii) $F_{1}(s)_{+}$is also an unknown function to be evaluated. The assumption here is that $f_{1}(x)=0 \exp (-\epsilon x)$ as $x \rightarrow \infty$ and is integrable near the origin. These conditions imply that $F_{1}(s)_{+}$is analytic for $\operatorname{Re}(s)>-\epsilon$ and $0|s|^{-1+q}, q>0$ as $|s| \rightarrow \infty$.

The relevant solution of Eq. (3.6) is

$$
\Phi=A Y^{1 / 2} K_{1 / n+2}\left[\left(-s^{2}\right)^{1 / 2} \frac{2}{n+2} Y^{n+2 / 2}\right]
$$

where $s$ must be purely imaginary to satisfy the condition $\Phi \rightarrow 0$ as $Y \rightarrow \infty$. Differentiation of (3.7) yields

$$
\frac{d \Phi}{d Y}=-A\left(-s^{2}\right)^{1 / 2} Y^{n+1 / 2} K_{n+1 / n+2}\left[\left(-s^{2}\right)^{1 / 2} \frac{2}{n+2} Y^{n+2 / 2}\right] .
$$

Evaluating (3.7) and (3.8) at $Y=0$ and using the boundary conditions from (3.6) we can write 


$$
\begin{aligned}
\Phi(s, 0) & =\pi / 2 \frac{(1 / n+2)^{-1 / n+2}(-s)^{-1 / 2 n+4}}{\sin \pi(n+1 / n+2) \Gamma(1 / n+2)} A=F_{1}(s)_{+}, \\
\frac{d \Phi(s, 0)}{d Y} & =-\pi / 2 \frac{(1 / n+2)^{-(n+1 / n+2)}(-s)^{-1 / 2 n+4}}{\sin \pi(n+1 / n+2) \Gamma(1 / n+2)} A=\theta_{1}(s)_{+}+\theta_{2}(s)_{-} .
\end{aligned}
$$

Eliminating $A$ from these two equations, we find,

$$
a F_{1}(s)_{+}=\frac{\theta_{1}(s)_{+}+\theta_{2}(s)_{-}}{\left(-s^{2}\right)^{1 / n+2}},
$$

where $a$ is given in Eq. (3.3).

As it stands, Eq. (3.10) is valid only for purely imaginary $s$. In order to use the Wiener-Hcpf technique, an expression which is analytic in a strip parallel to the imaginary axis is desired. Consider the function $\left(-s^{2}\right)^{1 / n+2}, \operatorname{Re}(s)=0$ which is real and positive. How should this function be continued analytically into the complex $s$-plane? Consider the product $(-s)^{1 / n+2}(s)^{1 / n+2}$ for complex $s$. $(-s)^{1 / n+2}$ is defined to be real when $s$ is real and negative and a cut is introduced in the $s$ plane along the positive real axis so that this function will be single-valued. $s^{1 / n+2}$ is defined to be real when $s$ is real and positive and a cut is introduced along the negative real axis. It is easily shown that when $s$ is imaginary this product is equal to $\left(-s^{2}\right)^{1 / n+2}$. The above product is taken as the continuation of $\left(-s^{2}\right)^{1 / n+2}$. Ncw let a small separation of the branch points be made by writing $(-s)^{1 / n+2}(s+\epsilon)^{1 / n+2}, \epsilon>0$. See Fig. 1. This is the same $\epsilon$ introduced in (i).

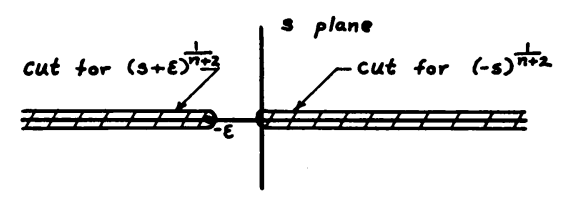

FIG. 1. Analytic region of $(-s)^{1 / n+2}(s+\epsilon)^{1 / n+2}$.

If this function is used in place of $\left(-s^{2}\right)^{1 / n+2}$ in (3.10) we get

$$
a F_{1}(s)_{+}(s+\epsilon)^{1 / n+2}=\frac{\Theta_{1}(s)_{+}+\Theta_{2}(s)_{-}}{(-s)^{1 / n+2}} .
$$

It is assumed that the solutions of this equation will satisfy (3.10) when $\epsilon$ goes to zero. This idea was used in [9]. Now the Weiner-Hopf technique can be used since both sides of Eq. (3.11) are analytic in the strip $-\epsilon<\operatorname{Re}(s)<0$.

We want to express $\theta_{1}(s)_{+} /(-s)_{-}^{1 / n+2}$ as the sum of two functions, one analytic in the left half plane, the other analytic in the overlapping right half plane. By Cauchy's Integral Formula [11],

$$
\begin{aligned}
\frac{\theta_{1}(s)_{+}}{(-s)^{1 / n+2}} & =\frac{1}{2 \pi i} \int_{\gamma_{2}-i \infty}^{\gamma_{2}+i \infty} \frac{1}{\omega-s} \frac{\theta_{1}(\omega)_{+}}{(-\omega)^{1 / n+2}} d \omega \\
& -\frac{1}{2 \pi i} \int_{\gamma_{1}-i \infty}^{\gamma_{1}+i \infty} \frac{1}{\omega-s} \frac{\theta_{1}(\omega)_{+}}{(-\omega)^{1 / n+2}} d \omega,
\end{aligned}
$$

where $-\epsilon<\gamma_{1}<\operatorname{Re}(s)<\gamma_{2}<0$. The first integral in Eq. (3.12) is analytic for $\operatorname{Re}(s)<\gamma_{2}$, while the second is analytic for $\operatorname{Re}(s)>\gamma_{1}$. Equation (3.12) can be 
written as

$$
\frac{\Theta_{1}(s)_{+}}{(-s)^{1 / n+2}}=\sigma_{2}(s)_{-}-\sigma_{1}(s)_{+},
$$

where $\sigma_{2}(s)_{-}, \sigma_{1}(s)_{+}$are defined by Eq. (3.12). Using Eq. (3.13), Eq. (3.11) becomes

$$
a f_{1}(s)_{+}(s+\epsilon)^{1 / n+2}+\sigma_{1}(s)_{+}=\frac{\theta_{2}(s)_{-}}{(-s)^{1 / n+2}}+\sigma_{2}(s)_{-}=E(s) .
$$

Since the two sides of this equation are analytic in overlapping half planes, they are sufficient to define an entire function $E(s)$. That is to say, the right hand side is the analytic continuation of the left hand side. From the estimates made in (i), (ii), (iii) and since $\gamma_{1}$ and $\gamma_{2}$ are of the order of $|s|^{-1}$, it is seen that

$$
\begin{aligned}
& E(s)_{+}=0\left(|s|^{(1 / n+2)-1+q}\right), \\
& E(s)_{-}=0\left(|s|^{(-1 / n+2)-1+p}\right),
\end{aligned}
$$

as $|s| \rightarrow \infty$, where $p, q<1$. The derivative of $E$ is bounded and goes to zero for large $s$. Therefore, by Liouvilles' theorem [11] $E$ is a constant. But the above shows $E(s)_{-} \rightarrow 0$ as $|s| \rightarrow \infty$. This implies that $E=0$. This fact allows the evaluation of $F_{1}(s)_{+}$. From Eq. (3.14),

$$
F_{1}(s)_{+}=-a^{-1} \frac{\sigma_{1}(s)_{+}}{(s+\epsilon)^{1 / n+2}}, \quad R e(s)>-\epsilon .
$$

Let $\epsilon \rightarrow 0$ and substitute the integral expression for $\sigma_{1}(s)_{+}$. This gives

$$
F_{1}(s)=a^{-1} s^{-1 / n+2} \frac{1}{2 \pi i} \int_{-i \infty}^{i \infty} \frac{1}{s-\omega} \frac{\theta_{1}(\omega)_{+}}{(-\omega)^{1 / n+2}} d \omega, \quad \operatorname{Re}(s)>0,
$$

where $\gamma_{1}=0$ since $\epsilon>\gamma_{1}>0$.

Taking the inverse transform of (3.16) we obtain

$$
\phi(x, 0)=f_{1}(x)=a^{-1} L^{-1}\left\{s^{-1 / n+2} \frac{1}{2 \pi i} \int_{-i \infty}^{i \infty} \frac{1}{s-\omega} \frac{\theta_{1}(\omega)_{+}}{(-\omega)^{1 / n+2}} d \omega\right\} .
$$

The inverse transform ${ }^{2}$ on the right hand side is the convolution of

$$
L^{-1}\left(s^{-1 / n+2}\right)=\frac{x^{(1 / n+2)-1} H(x)}{\Gamma(1 / n+2)}
$$

with

$$
L^{-1}\left\{\frac{1}{2 \pi i} \int_{-i \infty}^{i \infty} \frac{1}{s-\omega} \frac{\theta_{1}(\omega)_{+}}{(-\omega)^{1 / n+2}} d \omega\right\}=H(x) \frac{1}{2 \pi i} \int_{-i \infty}^{i \infty} e^{\omega x} \frac{\theta_{1}(\omega)_{+}}{(-\omega)^{1 / n+2}} d \omega \equiv H(x) \psi(x),
$$

where $\psi(x)$ is defined by this equation. Use of the convolution integral (the inverse transform of the product of two transforms) gives

$$
f_{1}(x)=a^{-1} \frac{1}{\Gamma(1 / n+2)} \int_{0}^{x}(x-\xi)^{(1 / n+2)-1} \psi(\xi) d \xi, \quad x>0
$$

2All Laplace and Mellin transforms used in this paper can be found in [10]. 
But $\psi(\xi)$ is the convolution of

$$
\frac{1}{2 \pi i} \int_{-i \infty}^{i \infty} e^{\omega \xi} \theta_{1}(\omega)+d \omega=\theta_{1}(\xi) H(\xi)
$$

with

$$
\frac{1}{2 \pi i} \int_{-i \infty}^{i \infty} e^{\omega \xi}(-\omega)^{-1 / n+2} d \omega=\frac{(-\xi)^{1 / n+2} H(-x)}{\Gamma(1 / n+2)}
$$

Therefore,

$$
\psi(\xi)=\frac{1}{\Gamma(1 / n+2)} \int_{\xi}^{\infty} \theta(\eta)(\eta-\xi)^{(1 / n+2)-1} d \eta, \quad \xi>0 .
$$

Substitution of Eq. (3.19) into Eq. (3.18) yields Eq. (3.3) as was to be shown.

4. The case where $\partial \phi(x, 0) / \partial y$ is given on $(0,1)$. The result of Sec. 3 is

$$
\begin{aligned}
& \phi(x, 0)=\frac{a^{-1}}{\Gamma^{2}(1 / n+2)} \int_{0}^{x}(x-\xi)^{(1 / n+2)-1} d \xi \int_{\xi}^{\infty} \theta_{1}(\eta)(\eta-\xi)^{(1 / n+2)-1} d \eta, \\
& 0<x<\infty \text {, }
\end{aligned}
$$

where $\theta_{1}(x)=\partial \phi(x, 0) / \partial y$ is supposed to be known on $0<x<\infty$. In the problem stated in Sec. $2, \partial \phi(x, 0) / \partial y=\theta(x)$ is specified only for the interval $(0,1)$ while $\phi(x, 0)$ is specified for $x>1$. Hence Eq. (4.1) is an integral equation for the unknown functions. It will be shown that if

$$
\frac{\partial \phi(x, 0)}{\partial Y}=\theta_{1}(x)=\theta(x) H(1-x)+\theta_{3}(x) H(x-1), \quad x>0
$$

and

$$
\phi(x, 0)=f(x) H(1-x)-\frac{C}{2} H(x-1), \quad x>0
$$

where $f(x)$ and $\theta_{3}(x)$ are unknown functions, then the desired solution of Eq. (4.1) is

$$
\begin{aligned}
& \phi(x, 0)=f(x)=-\frac{C}{2} \frac{\Gamma(2 / n+2)}{\Gamma^{2}(1 / n+2)} \int_{0}^{x} \xi^{(1 / n+2)-1}(1-\xi)^{(1 / n+2)-1} d \xi \\
& +\frac{a^{-1} x^{1 / n+2}}{\Gamma^{2}(1 / n+2)} \int_{x}^{1}(\xi-x)^{(1 / n+2)-1} \xi^{-2 / n+2} d \xi \int_{0}^{\xi}(\xi-\eta)^{(1 / n+2)-1} \eta^{1 / n+2} \theta(\eta) d \eta,
\end{aligned}
$$

when $x$ is between 0 and 1 .

To prove Eq. (4.3) the same procedure is followed as in Sec. 3, using the Mellin transform in place of the Laplace transform. Operating on both sides of (4.1) with the Mellin transform, defined by

$$
M_{s}[f(x)]=\int_{0}^{\infty} x^{s-1} f(x) d x,
$$

yields the relation ${ }^{3}$

$$
a M_{s}[\phi(x, 0)]=\frac{\Gamma[1-s-(1 / n+2)] \Gamma[s+(1 / n+2)]}{\Gamma(1-s) \Gamma[s+(2 / n+2)]} M_{\bullet}\left[x^{2 / n+2} \theta_{1}(x)\right] .
$$

SThis is the Mellin transform of a repeated fractional integral. See [10] , vol. 2, p. 183. 
A similar operation on Eq. (4.2) yields

$$
\begin{aligned}
M_{s}[\phi(x, 0)] & =M_{s}[f(x) H(1-x)]-\frac{C}{2} M_{s}[H(x-1)]=F(s)_{+}+\frac{C}{2} \frac{1}{s_{-}}, \\
M_{s}\left[x^{2 / n+2} \theta_{1}(x)\right] & =M_{s}\left[x^{2 / n+2} \theta(x) H(1-x)\right]+M_{s}\left[x^{2 / n+2} \theta_{3}(x) H(x-1)\right] \\
& =T(s)_{+}+T_{3}(s)_{-},
\end{aligned}
$$

where

$$
\begin{gathered}
F(s)_{+}=\int_{0}^{1} x^{s-1} f(x) d x, \\
\frac{1}{s_{-}}=-M_{s}[H(x-1)], \quad \operatorname{Re}(s)<0, \\
T(s)_{+}=\int_{0}^{1} x^{s-1} x^{2 / n+2} \theta(x) d x, \\
T_{3}(s)_{-}=\int_{1}^{\infty} x^{s-1} x^{2 / n+2} \theta_{3}(x) d x .
\end{gathered}
$$

Substitution of Eq. (4.5) into Eq. (4.4) yields

$$
a F(s)_{+}+a \frac{C}{2} \frac{1}{s_{-}}=\frac{\Gamma[1-s-(1 / n+2)]}{\Gamma(1-s)} \frac{\Gamma[s+(1 / n+2)]}{\Gamma[s+(2 / n+2)]}\left[T(s)_{+}+T_{3}(s) .\right] .
$$

As in Sec. 3, the regions of analyticity and the asymptotic behavior of $F, T, T_{3}$ are required and are shown as follows:

(i) $F(s)_{+}$is an unknown function. It is assumed $f(x)=0\left(x^{1 / n+2}\right)$ as $x \rightarrow 0$; then $F(s)_{+}$ is analytic for $\operatorname{Re}(s)>-1 / n+2$. In order to determine the asymptotic behavior of $F(s)_{+}$, substitute $x=\exp (-z)$ into the defining integral above, so that it takes the form of a Laplace integral. It is easily seen that if $f(x)=0|1-x|^{-p}, p<1$ as $x \rightarrow 1$ then $F(s)_{+}=0|s|^{-1+p}$ as $|s| \rightarrow \infty$. This assumption is necessary in order that $F(s)_{+}$ exist.

(ii) $T(s)_{+}$is a known function. Since $\theta(x)$ is a known bounded function, $T(s)_{+}$is analytic for $\operatorname{Re}(s)>-2 / n+2$. The substitution $x=\exp (-z)$ shows that $T(s)=$ $0|s|^{-1}$ as $|s| \rightarrow \infty$.

(iii) $T_{3}(s)_{-}$is an unknown function. By comparison with known potential solutions (i.e., potential flow about a flat plate with circulation) it is assumed that the unknown function $\theta_{3}(x)=0\left(x^{-2 / n+2}\right)$ as $x \rightarrow \infty$ so that $T_{3}(s)_{-}$is analytic for $\operatorname{Re}(s)<0$. If it is assumed that $\theta_{3}(x)=0(x-1)^{-a}, q<1$ as $x \rightarrow 1$, the substitution $x=e^{z}$ obtains $T_{3}(s)_{-}=$ $0|s|^{-1+a}$ as $|s| \rightarrow \infty$.

(iv) The asymptotic behavior of ratios of gamma functions in Eq. (4.6) may be found by substituting $x=\exp (-\sigma)$ into the beta function representation,

$$
\frac{\Gamma(s)}{\Gamma(s+\nu)}=\frac{1}{\Gamma(\nu)} \int_{0}^{1} x^{s-1}(1-x)^{\nu-1} d x .
$$

From this it is found that the function $\Gamma[s+(2 / n+2)] / \Gamma[s+(1 / n+2)]$ which is analytic for $\operatorname{Re}(s)<1-(1 / n+2)$ is of the order $|s|^{1 / n+2}$ as $|s| \rightarrow \infty$, while $\Gamma[1-s-(1 / n+2)] / \Gamma(1-s)$ is analytic for $\operatorname{Re}(s)<1-(1 / n+2)$ and is of the order $|s|^{-1 / n+2}$ as $|s| \rightarrow \infty$. 
Rearrangement of Eq. (4.6) gives

$$
\begin{aligned}
a F(s)_{+} \frac{\Gamma[s+(2 / n+2)]}{\Gamma[s+(1 / n+2)]}=\frac{\Gamma[1-s-(1 / n+2)]}{\Gamma(1-s)} T_{3}(s)_{-} \\
-a \frac{C}{2} \frac{1}{s_{-}} \frac{\Gamma[s+(2 / n+2)]}{\Gamma[s+(1 / n+2)]}+T(s)_{+} \frac{\Gamma[1-s-(1 / n+2)]}{\Gamma(1-s)} .
\end{aligned}
$$

A little reflection on (i), (ii), (iii) and (iv) shows that all the functions occuring in Eq. (4.7) are analytic in the strip $-(1 / n+2)<\operatorname{Re}(s)<0$. As in Sec. 3, we can write

$$
\begin{gathered}
\frac{\Gamma[1-s-(1 / n+2)]}{\Gamma(1-s)} T(s)_{+}=\frac{1}{2 \pi i} \int_{\beta_{2}-i \infty}^{\beta_{2}+i \infty} \frac{1}{\omega-s} \frac{\Gamma[1-\omega-(1 / n+2)]}{\Gamma(1-\omega)} T(\omega)_{+} d \omega \\
-\frac{1}{2 \pi i} \int_{\beta_{1}-i \infty}^{\beta_{1}+i \infty} \frac{1}{\omega-s} \frac{\Gamma[1-\omega-(1 / n+2)]}{\Gamma(1-\omega)} T(\omega)_{+} d \omega=\lambda_{2}(s)_{-}-\lambda_{1}(s)_{+},
\end{gathered}
$$

where $-(1 / n+2)<\beta_{1}<\operatorname{Re}(s)<\beta_{2}<0$ and $\lambda_{2}(s)_{-}$and $\lambda_{1}(s)_{+}$represent the first and the second integral on the right hand side. $\lambda_{2}(s)_{-}$is analytic for $\operatorname{Re}(s)<\beta_{2}$ while $\lambda_{1}(s)_{+}$is analytic for $\operatorname{Re}(s)>\beta_{1}$. Also, by subtracting the principal part of the singularity at $s=0$

$\frac{1}{s} \frac{\Gamma[s+(2 / n+2)]}{\Gamma[s+(1 / n+2)]}=\left\{\frac{1}{s} \frac{\Gamma[s+(2 / n+2)]}{\Gamma[s+(1 / n+2)]}-\frac{1}{s} \frac{\Gamma(2 / n+2)}{\Gamma(1 / n+2)}\right\}_{+}+\frac{1}{s_{-}} \frac{\Gamma(2 / n+2)}{\Gamma(1 / n+2)}$.

The bracketed term on the right is analytic for $\operatorname{Re}(s)>-2 / n+2$, while the second term on the right is analytic for $\operatorname{Re}(s)<0$. Introducing the results of Eq. (4.8) and Eq. (4.9) into Eq. (4.7) gives

$$
\begin{aligned}
E_{1}(s)=a F(s)_{+} & \frac{\Gamma[s+(2 / n+2)]}{\Gamma[s+(1 / n+2)]}+\lambda_{1}(s)_{+} \\
& +a \frac{C}{2}\left\{\frac{1}{s} \frac{\Gamma[s+(2 / n+2)]}{\Gamma[s+(1 / n+2)]}-\frac{1}{s} \frac{\Gamma(2 / n+2)}{\Gamma(1 / n+2)}\right\}_{+} \\
= & \frac{\Gamma[1-s-(1 / n+2)]}{\Gamma(1-s)} T_{3}(s)_{-}+\lambda_{2}(s)_{-}-a \frac{C}{2} \frac{\Gamma(2 / n+2)}{\Gamma(1 / n+2)} \frac{1}{s_{-}} .
\end{aligned}
$$

As in Sec. 3, since the left and right sides of this equation are analytic in overlapping half planes, an entire function $E_{1}(s)$ can be defined. From the estimates in (i), (ii), (iii) and (iv) it is found that

$$
E_{1}(s)_{+}=0|s|^{(1 / n+2)-1+p}
$$

and

as

$$
E_{1}(s)_{-}=0|s|^{-(1 / n+2)-1+q}
$$

$$
|s| \rightarrow \infty, \text { where } p, q<1 \text {. }
$$

These estimates imply $E_{1}(s) \equiv 0$. This determines $F(s)_{+}$and $T_{3}(s)_{-}$in Eq. (4.10) as follows

$$
\begin{aligned}
F(s)_{+}=- & a^{-1} \frac{\Gamma[s+(1 / n+2)]}{\Gamma[s+(2 / n+2)]} \lambda_{1}(s)_{+} \\
& -\frac{C}{2}\left\{\frac{1}{s}-\frac{1}{s} \frac{\Gamma(2 / n+2)}{\Gamma(1 / n+2)} \frac{\Gamma[s+(1 / n+2)]}{\Gamma[s+(2 / n+2)]}\right\}_{+} \quad \operatorname{Re}(s)>-1 / n+2
\end{aligned}
$$




$$
\begin{aligned}
T_{3}(s)_{-}=-\frac{\Gamma(1-s)}{\Gamma[1-s-(1 / n+2)]} \lambda_{2}(s)_{-} \\
\quad+a \frac{C}{2} \frac{\Gamma(2 / n+2)}{\Gamma(1 / n+2)} \frac{1}{s} \frac{\Gamma(1-s)}{\Gamma[1-s-(1 / n+2)]} \operatorname{Re}(s)<0 .
\end{aligned}
$$

The next step is to take the inverse Mellin transform of Eq. (4.12) using the Mellin convolution in the same way that we used the Laplace convolution in Sec. 3. The result is Eq. (4.3) which was to be demonstrated.

5. Solution of the mixed boundary value problem stated in Sec. 1. The original problem to be solved was a mixed boundary value problem. Since from Sec. $4, \phi(x, 0)$ is known on the interval $(0,1)$, we know $\phi(x, 0)$ for the infinite interval. This reduces the mixed boundary value problem to a much simpler Dirichlet problem.

$$
\begin{aligned}
& Y^{n} \frac{\partial^{2} \phi}{\partial x^{2}}+\frac{\partial^{2} \phi}{\partial Y^{2}}=0, \quad Y \geqq 0, \quad n \geqq 0 ; \\
& \phi(x, 0)=\phi_{0}(x), \quad-\infty<x<\infty ; \\
& \phi(x, \infty)=0 .
\end{aligned}
$$

Here

$$
\phi_{0}(x)=\left\{\begin{array}{cl}
0 & x<0 \\
f(x) & 0<x<1 \\
-C / 2 & x>1
\end{array}\right.
$$

where $f(x)$ is defined by Eq. (4.3). Use of Laplace transforms as in Eq. (3.6) yields the bounded solution shown in Eq. (3.7). From Eq. (3.9)

$$
\Phi(s, 0)=\frac{\pi}{2} \frac{(-s)^{-1 / 2 n+4}}{(1 / n+2)^{1 / n+2} \sin (\pi / n+2) \Gamma(n+1 / n+2)} A=\Phi_{0}(s),
$$

where $\Phi_{0}(s)$ is the two-sided Laplace integral of $\phi_{0}(x)$. Solving Eq. (5.2) for $A$ and substituting it into Eq. (3.7),

$$
\begin{aligned}
\Phi=\frac{2}{\pi}(1 / n+2)^{1 / n+2} \sin (\pi / n+2) \Gamma(n+1 / n+2)\left(-s^{2}\right)^{1 / 2 n+4} \\
\cdot Y^{1 / 2} K_{1 / n+2}\left[\left(-s^{2}\right)^{1 / 2} \frac{2}{n+2} Y^{n+2 / 2}\right] \Phi_{0} \quad \operatorname{Re}(s)=0 .
\end{aligned}
$$

The inversion of this gives the result

$$
\phi(x, Y)=d_{n} \int_{-\infty}^{\infty} \frac{\phi_{0}(\xi) Y d \xi}{\left[(x-\xi)^{2}+(2 / n+2) Y^{n+2}\right]^{(1 / n+2)+1 / 2}},
$$

where $d_{n}=\pi^{-3 / 2}(2 / n+2)^{2 / n+2} \Gamma[(1 / n+2)+1 / 2] \Gamma(n+1 / n+2) \sin (\pi / n+2)$. Since this result will not be used in Part II, no further comment will be made.

Part II. Airfoil in a Sonic Shear Flow Jet

6. Solution of shear flow problem. As seen in Sec. 1, for a symmetrical Mach 
number distribution the potential $\phi$ must satisfy

$$
\left(1-M^{2}\right) \frac{\partial^{2} \phi}{\partial x^{2}}+\frac{\partial^{2} \phi}{\partial y^{2}}-\frac{2}{M} \frac{d M}{d y} \frac{\partial \phi}{\partial y}=0
$$

for $y \geqq 0$, and the boundary conditions

$$
\begin{aligned}
\phi(x, 0) & =0 & & x<0, \\
& =-C_{l / 2} & & x>1, \\
\frac{\partial \phi(x, 0)}{\partial y} & =-2 \alpha_{0}(x) & & 0<x<1, \\
\phi(x, \infty) & =0 . & &
\end{aligned}
$$

Introducing the new independent variable

$$
Y=b \int_{0}^{y} M^{2}(y) d y
$$

where $b$ is a constant, Eq. (7.1) is transformed into

$$
\frac{1-M^{2}}{b^{2} M^{4}} \frac{\partial^{2} \phi}{\partial x^{2}}+\frac{\partial^{2} \phi}{\partial Y^{2}}=0 \text {. }
$$

Let $M(y)$, an arbitrary function until now, be defined by

$$
\frac{1-M^{2}}{b^{2} M^{4}}=Y^{n}=\left(b \int_{0}^{y} M^{2} d y\right),
$$

where $n$ is a non-negative number, so that Eq. (6.4) becomes the generalized Tricomi equation treated in Part I. The solution of (6.5), which gives the Mach number distribution, will be investigated in Sec. 7. The transformation by Eq. (6.3) also changes the third equation in the boundary conditions, Eq. (6.2) to

$$
\frac{\partial \phi(x, 0)}{\partial Y}=-\frac{2 \alpha_{0}(x)}{b M_{0}^{2}}, \quad 0<x<1 .
$$

It is seen immediately that this is the problem solved in Part I with the notational change $\theta(x)=-2 \delta_{0}(x) / b M_{0}^{2}$ and $C=C_{l}$. The solution of this problem involves the constant $C_{l}$ which has been presumed specified in advance. To determine $C_{l}$ the KuttaJoukowsky condition should be used. This condition states that the flow must leave the trailing edge smoothly. In a linearized approach, this means that the streamline leaving the trailing edge of the airfoil should have finite slope at the trailing edge. In Appendix II it is shown that the slope is finite provided that

$C_{l}=\frac{2 \Gamma(1 / n+2)}{\Gamma(2 / n+2)} \frac{(1 / n+2)^{n / n+2}}{\Gamma(n+1 / n+2)} \int_{0}^{1}(1-\eta)^{(1 / n+2)-1} \eta^{1 / n+2}\left[-2 \alpha_{0}(\eta) / b M_{0}^{2}\right] d \eta$.

This gives the lift coefficient in terms of the airfoil slope. The pressure coefficient on the airfoil can be calculated by taking the $x$ derivative of Eq. $(4.3)$, since $C_{p}(x, 0)=$ $\partial \phi(x, 0) / \partial x$. This may be accomplished more easily if the integration variable in the second integral in Eq. (4.3) is changed by the substitution $z=x / \xi$. The result is 


$$
\begin{aligned}
C_{p}(x, 0)=e_{n} \int_{1}^{x}(1-z)^{(1 / n+2)-1} z^{(1 / n+2)-1} d z \frac{\partial}{\partial x} \\
\cdot \int_{0}^{x / z}\left(\frac{x}{z}-\eta\right)^{(1 / n+2)-1} \eta^{1 / n+2}\left[-2 \alpha_{0}(\eta) / b M_{0}^{2}\right] d \eta
\end{aligned}
$$

where

$$
e_{n}=\frac{-(1 / n+2)^{n / n+2}}{\Gamma(n+1 / n+2) \Gamma(1 / n+2)} .
$$

It will be shown in Sec. 8 that Fq. (6.8) can be integrated with a simple result if $\alpha_{0}(x)$ is a polynomial.

7. Mach number profile. Certain properties of the upstream Mach number distribution, $M(y)$, which are defined by the solution of Eq. (6.5), can easily be seen without completely solving this equation. These will be enumerated below.

(i) If $n=0, M=M_{0}$ is constant and less than one. In this case $b(n=0)=b_{0}$ can be expressed in terms of $M_{0}$ as $b_{6}=\left(1-M_{0}^{2}\right)^{1 / 2} / M_{0}^{2}$. Noticing how $b_{0}$ occurs in Eq. (6.8), it is seen that $b_{0}$ is essentially the Prandtl-Glauert correction factor for incompressible flow.

(ii) For $n=0, M \rightarrow 1$ as $y \rightarrow 0$ while $M \rightarrow 0$ as $y \rightarrow \infty$.

(iii) To see how $M$ behaves near $y=0$, rewrite Eq. (6.5) as

$$
b^{(n+2) / n} \int_{0}^{\nu} M^{2} d y=\left(\frac{1-M^{2}}{M^{4}}\right)^{1 / n}
$$

and substitute a series, $M=1-y^{\nu}\left(a_{\iota}+a_{1} y+\cdots\right)$, for $M$. Solving the resulting equation for $a_{0}$ and $\nu$ it is found that

$$
M=1-\frac{1}{2} b^{n+2} y^{n}+\cdots .
$$

This shows that in addition to being sonic at $y=0$, the profile becomes increasingly flat near $y=0$ as $n$ increases. This is of some interest since, as is well known, purely sonic uniform flow cannot be linearized. Notice also that $b \rightarrow 0$ implies uniform sonic flow, while from Eq. (6.8) the pressure becomes infinite as $b \rightarrow 0$, so that the linearization has become invalid.

(iv) The behavior for small $n$ can also be seen from Eq. (7.2). If $0<n<1, M$ has a cusp at $y=0$ which becomes sharper as $n$ decreases. Upon taking the limit as $n \rightarrow 0$ the cusp collapses on itself leaving uniform subsonic flow.

Since the solution of Eq. (7.1) for $M$ as a function of $y$ is difficult to obtain explicitly, the inverse of this function $y=y(M)$ will be considered. Denoting $M^{2}$ by $\xi$ and differentiating Eq. (7.1) with respect to $y$ we get

$$
b^{(n+2) / n} \xi=\frac{d}{d \xi}\left(\frac{1-\xi}{\xi^{2}}\right)^{1 / n} \frac{d \xi}{d y} .
$$

Upon separating variables and integrating,

$$
b^{(n+2) / n} y=\int_{1}^{M M^{2}} \frac{1}{\xi} \frac{d}{d \xi}\left(\frac{1-\xi}{\xi^{2}}\right)^{1 / n} d \xi
$$

is obtained. An integration by parts gives

$$
b^{(n+2) / n} y=\frac{1}{M^{2}}\left(\frac{1-M^{2}}{M^{4}}\right)^{1 / n}-\int_{M^{2}}^{1} \frac{1}{\xi^{2}}\left(\frac{1-\xi}{\xi^{2}}\right)^{1 / n} d \xi .
$$


This is the desired inverse function. If $n$ equals 1 or 2 the integration can be carried out explicitly. For large $n$ a series in $1 / n$ is appropriate. The results of these calculations are given in Fig. 2. In this figure $M$ is plotted versus $y b^{(n+2) / n}$. If $b=1$ the various curves are in the correct proportions as plotted, however, if $b \neq 1$ the scale changes for each curve. If $n$ is very large the profiles are again approximately in the proper proportions. Note that as $n \rightarrow \infty$ the profile approaches a uniform sonic jet of width $2 / b$; twice the reciprocal of $b$ can be considered roughly to be an effective jet width.

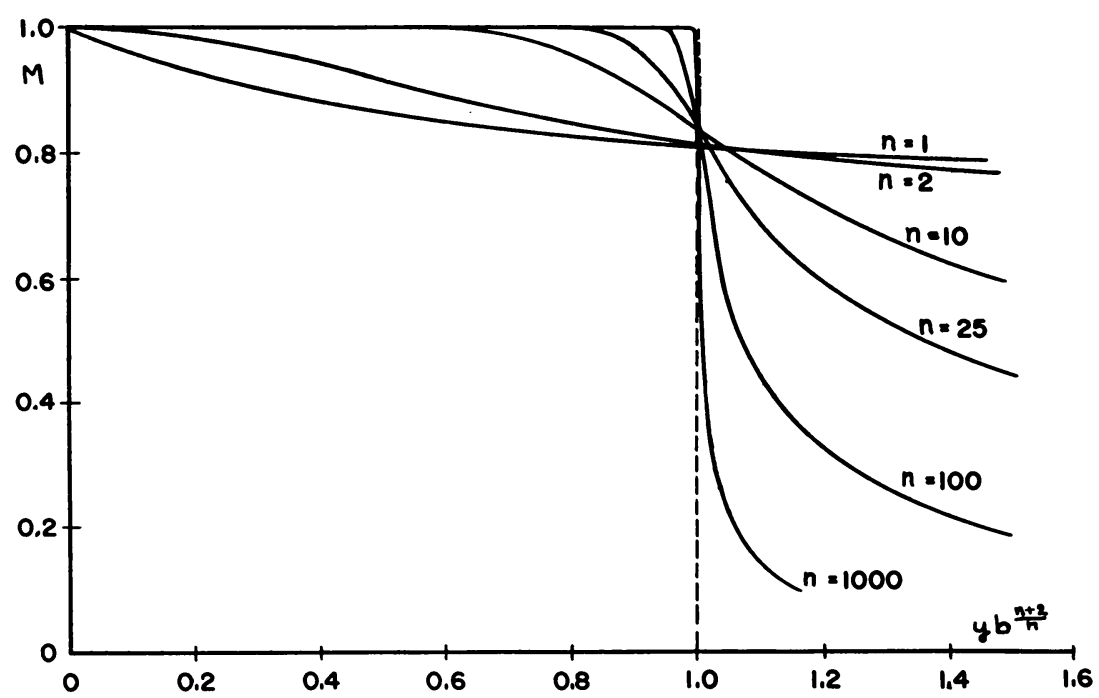

FIg. 2. Mach number versus vertical distance with $n$ as parameter.

8. Calculations of $C_{p}, C_{l}$ and C.P. In this section the calculation of the pressure coefficient, lift coefficient and center of pressure (C.P.) will be carried out for the case where $\alpha_{0}(x)$ is a polynomial. In this case $-2 \alpha_{0}(x) / b M_{0}^{2}$ can be expressed as a sum of terms like $a_{i} x^{i}$ and the lift coefficient, Eq. (6.7) will be a sum of terms like

$$
C_{l j}=2 a_{i} \frac{\Gamma(1 / n+2)(1 / n+2)^{n / n+2}}{\Gamma(2 / n+2) \Gamma(n+1 / n+2)} \int_{0}^{1}(1-\eta)^{(1 / n+2)-1} \eta^{i+(1 / n+2)} d \eta .
$$

This integral is a beta function which is easily expressed as

$$
C_{l i}=2 a_{i} \frac{\Gamma(1 / n+2)(1 / n+2)^{n / n+2}}{\Gamma(2 / n+2) \Gamma(n+1 / n+2)} \frac{\Gamma(1 / n+2) \Gamma[(1 / n+2)+j+1]}{\Gamma[(2 / n+2)+j+1]} .
$$

The pressure coefficient, Eq. (6.8), on the airfoil will be a sum of terms like

$$
C_{p i}=g_{n} \int_{x}^{1}(1-z)^{(1 / n+2)-1} z^{(1 / n+2)-1} d z \frac{\partial}{\partial x} \int_{0}^{z / z}\left(\frac{x}{z}-\eta\right)^{(1 / n+2)-1} \eta^{j+(1 / n+2)} d \eta
$$

where

$$
g_{n}=-\frac{a_{j}(1 / n+2)^{n / n+2}}{\Gamma(n+1 / n+2) \Gamma(1 / n+2)} .
$$

The inner integral, a fractional integral of the Riemann-Liouville type can be evaluated 
as

$$
\int_{0}^{x / z}\left(\frac{x}{z}-\eta\right)^{(1 / n+2)-1} \eta^{j+(1 / n+2)} d \eta=\Gamma(1 / n+2) \frac{\Gamma[j+1+(1 / n+2)]}{\Gamma[j+1+(2 / n+2)]}(x / z)^{j+(2 / n+2)} .
$$

Then

$$
C_{p i}=k_{n} \int_{x}^{1}(1-z)^{(1 / n+2)-i-1} z^{-(1 / n+2)-i-1} d z
$$

where

$$
k_{n}=-\frac{a_{j}(1 / n+2)^{n / n+2}}{\Gamma(n+1 / n+2)} \frac{\Gamma[j+1+(1 / n+2)]}{\Gamma[j+1+(2 / n+2)]} x^{j-1+(2 / n+2)} .
$$

By a change of the variable of integration, $t=(1-z) / z$ this becomes

$$
\begin{aligned}
C_{p i}=-a_{i} \frac{(1 / n+2)^{n / n+2}}{\Gamma(n+1 / n+2)} \frac{\Gamma[j+1+(1 / n+2)]}{\Gamma[j+1+(2 / n+2)]} x^{i-1+(2 / n+2)} \\
\cdot \int_{0}^{(1-x) / x} t^{(1 / n+2)-1}(1+t)^{i} d t .
\end{aligned}
$$

The integration here can be easily carried out for any value of $j$ by expanding $(i+t)^{i}$.

Let a moment coefficient be defined by

$$
C_{m}=-2 \int_{0}^{1} x C_{p} d x
$$

This can be computed after $C_{p}$ is known. A more useful quantity which will be used is the center of pressure defined by

$$
\text { C.P. }=C_{m} / C_{l} \text {. }
$$

These quantities $\left(C_{l}, C_{p}, C . P.\right)$ are calculated below for the special cases of flat plate and circular arc airfoils.

(i) Flat plate airfoil at angle of attack $\delta$.

In this case, using the above notation, $\alpha_{0}(x)=-\delta$ is a constant. Then

$$
\frac{-2 \alpha_{0}(x)}{b M_{0}^{2}}=\frac{2 \delta}{b M_{0}^{2}}=a_{0} \text {. }
$$

From Eq. (8.1),

$$
C_{l}=\frac{2 \delta}{b M_{0}^{2}}\left(\frac{1}{n+2}\right)^{n / n+2} \frac{\Gamma^{3}(1 / n+2)}{\Gamma^{2}(2 / n+2) \Gamma(n+1 / n+2)} .
$$

If $n=0$ (uniform subsonic flow) this gives the familiar result

$$
C_{l}=\frac{2 \pi \delta}{b M_{0}^{2}}=\frac{2 \pi \delta}{\left(1-M^{2}\right)^{1 / 2}}
$$

where $b=\left(1-M^{2}\right)^{1 / 2} / M^{2}, M$ constant. If $n \rightarrow \infty$ (sonic jet) the result is $C_{\imath}=8 \delta / b=$ $4 h \delta$. Here the jet thickness $h=2 / b$ has been used. Equation (8.5) is plotted in Fig. 3. From Eq. (8.2) the pressure coefficient

$$
C_{p} / C_{\imath}=-\frac{\Gamma(2 / n+2)}{\Gamma^{2}(1 / n+2)} x^{(1 / n+2)-1}(1-x)^{1 / n+2}
$$




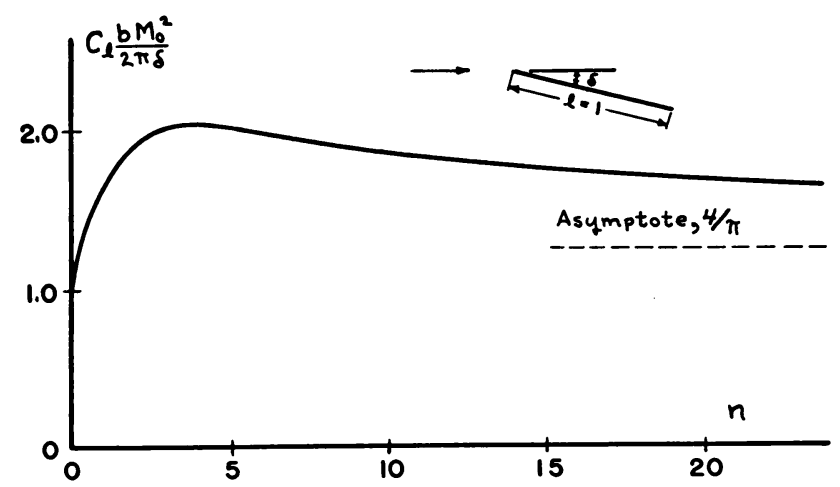

Fig. 3. Lift coefficient on flat plate versus $n$.

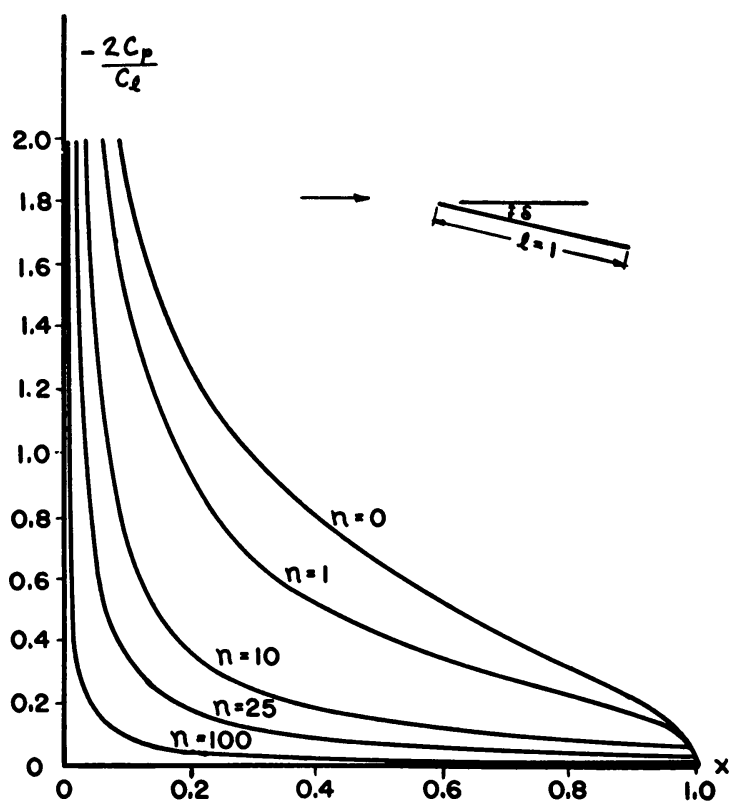

FIg. 4. Pressure coefficient on flat plate airfoil versus distance along airfoil.

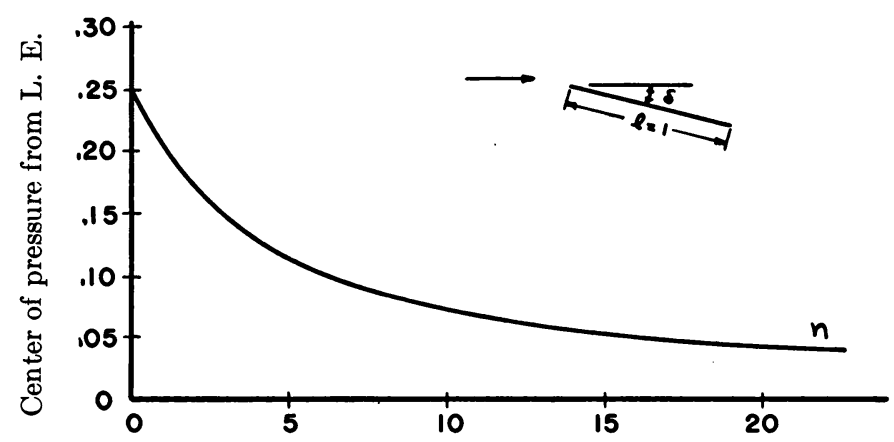

FIG. 5. Center of pressure on flat plate airfoil versus $n$. 


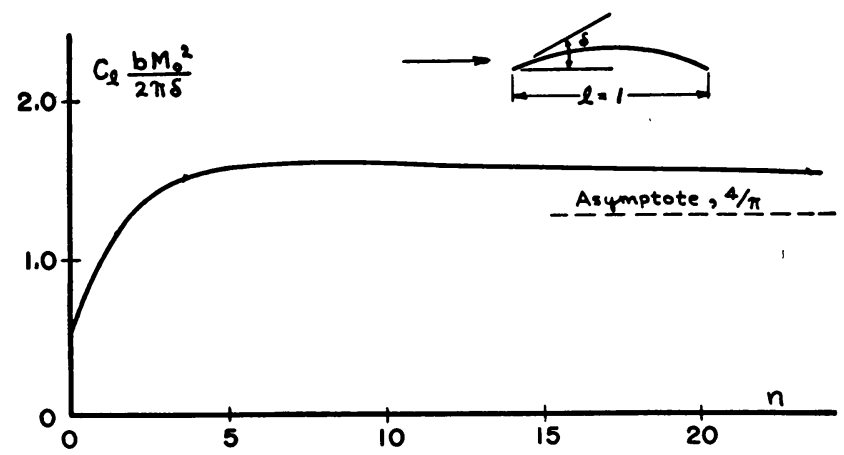

Fig. 6. Lift coefficient on circular arc airfoil versus $n$.

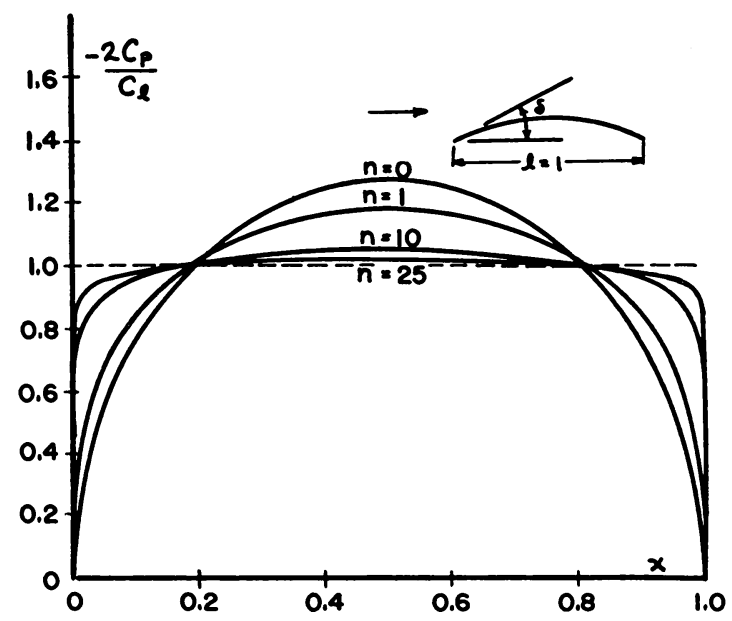

Fig. 7. Pressure coefficient on circular arc airfoil versus distance along airfoil.

is obtained. In Fig. 4, $-2 C_{p} / C_{l}$ has been plotted versus $x$ so that the "area" under each of the curves is unity. Notice that for $n=0$ the leading edge singularity is like $x^{-1 / 2}$. As $n$ becomes larger this singularity becomes stronger, approaching $x^{-1}$ as $n \rightarrow \infty$. The center of pressure is obtained by substituting Eq. (8.6) into Eq. (8.3). The result is the simple expression

$$
\text { C.P. }=\frac{1}{n+4} .
$$

This shows that for $n=0$ the center of pressure is at the quarter chord and approaches the leading edge as a $n$ increases. This is presented in Fig. 5 .

(ii) Circular arc airfoil at zero geometric angle of attack. For the circular arc $\alpha_{0}(x)=$ $\delta(1-2 x)$ where $\delta$ is the angle which the leading edge makes with the free stream. Then

$$
\frac{-2 \alpha_{0}(x)}{b M_{0}^{2}}=a_{0}+a_{1} x, \quad a_{0}=\frac{-2 \delta}{b M_{0}^{2}}, \quad a_{1}=\frac{4 \delta}{b M_{0}^{2}} .
$$

The lift coefficient and pressure coefficient are easily calculated from Eqs. (8.1) and 
(8.2). We obtain

$$
\begin{gathered}
C_{l}=\left(\frac{2 \delta}{b M_{0}^{2}}\right)(n+2 / n+4)(1 / n+2)^{n / n+2} \frac{\Gamma^{3}(1 / n+2)}{\Gamma^{2}(2 / n+2) \Gamma(n+1 / n+2)} \\
\frac{C_{p}}{C_{l}}=-\frac{\Gamma(2 / n+2)}{\Gamma^{2}(1 / n+2)}(n+4) x^{1 / n+2}(1-x)^{1 / n+2}
\end{gathered}
$$

The center of pressure is at $x=.5$ for all $n$ as can be seen from the symmetry of the pressure coefficient. Equations (8.8) and (8.9) are presented in Figs. 6 and 7. Note in Fig. 7 that as $n \rightarrow \infty$ the pressure coefficient becomes constant along the airfoil.

9. Discussion. It has been shown that for a particular class of Mach number profiles which are characterized by a sonic line, the linearized compressible shear flow equation can be transformed into the generalized Tricomi equation. The boundary value problem, which describes the perturbation of the main stream flow by an arbitrary two-dimensional camber surface, has been formulated and solved. The question of agreement with reality remains.

It is probable that for large values of $n$ the linearization breaks down unless restrictions are made on the parameter $b$, which should be large as $n$ becomes large. That is, the effective jet width, $2 / b$, must be much less than the chord length. This conjecture is supported by the following observation; by the foregoing theory the lift on an airfoil in the limiting case $n \rightarrow \infty$ is the same as that calculated by a simple momentum exchange brought about by the sonic jet turning through an angle equal to the trailing edge angle of the airfoil. Such a situation can only be realized if the jet width is much less than the chord length.

\section{APPENDIX I.}

Jump condition. Let $C$ denote the curve indicated in Fig. 8, consisting of two straight lines and a circle of radius $R$. If $\nabla \phi$ has continuous derivatives inside $C$ we can write

$$
\int_{c}\left(\frac{\partial \phi}{\partial x} d x+\frac{\partial \phi}{\partial y} d y\right)=0
$$

or

$$
\int_{0}^{R}\left[C_{p}\left(x, 0_{+}\right)-C_{p}\left(x, 0_{-}\right)\right] d x+\int_{0}^{2 \pi} \frac{\partial \phi}{\partial \theta} d \theta=0
$$

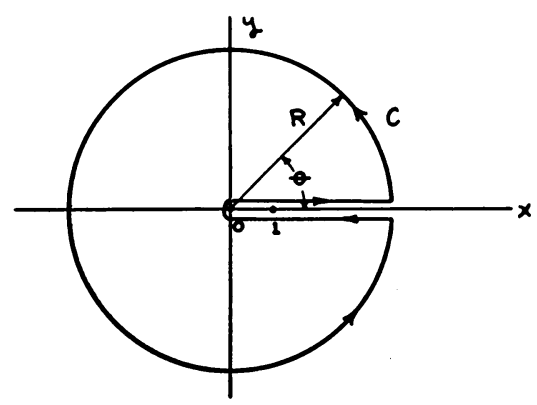

FIG. 8. 
Here $\partial \phi / \partial x=C_{p}$ has been used. $C_{p}$ must be continuous except across the airfoil itself, therefore $C_{p}\left(x, 0_{+}\right)-C_{p}\left(x, 0_{-}\right)$must be zero for $x>1$. Then the first integral is the negative of the lift coefficient,

$$
C_{l}=\int_{0}^{1}\left[C_{p}\left(x, 0_{-}\right)-C_{p}\left(x, 0_{+}\right)\right] d x=\frac{\text { Lift }}{(\gamma / 2) P M_{0}^{2}},
$$

while the second integral is $\phi\left(R, 0_{-}\right)-\phi\left(R, 0_{+}\right)$. Hence

$$
C_{l}=\phi\left(R, 0_{-}\right)-\phi\left(R, 0_{+}\right) \text {. }
$$

Since $C_{l}$ is independent of $R$ the jump across the $x$-axis must be constant.

\section{APPENDIX II.}

Kutta-Joukowsky condition. It is necessary that $\partial \phi(x, 0) / \partial Y$ be bounded as $x \rightarrow 1$ from the right. That is, $\theta_{3}(x)$ in Eq. (4.2) should be bounded. This condition will be satisfied only for a certain value of $C . \theta_{3}(x)$ can be found by inverting Eq. (4.13). Proceeding as with the inversion of Eq. (4.12) yields, after an integration by parts.

$$
\begin{gathered}
\theta_{3}(x)=\frac{x^{-1 / n+2}(x-1)^{-1 / n+2}}{\Gamma(n+1 / n+2) \Gamma(1 / n+2)}\left[-\frac{a C}{2} \Gamma(2 / n+2)\right. \\
\left.-\int_{0}^{1}(1-\eta)^{(1 / n+2)-1} \eta^{1 / n+2} \theta(\eta) d \eta\right]+\frac{(n+1 / n+2) x^{-1 / n+2}}{\Gamma(n+1 / n+2) \Gamma(1 / n+2)} \\
\cdot \int_{1}^{x} \xi^{2 / n+2}(x-\xi)^{-1 / n+2} d \xi \int_{0}^{1}(\xi-\eta)^{(1 / n+2)-2} \eta^{1 / n+2} \theta(\eta) d \eta .
\end{gathered}
$$

Therefore $\theta_{3}(x)$ will be bounded at $x=1$ if the coefficient of $(x-1)^{-1 / n+2}$ is zero, that is, if

$$
\frac{C}{2}=-\frac{a^{-1}}{\Gamma(2 / n+2)} \int_{0}^{1}(1-\eta)^{(1 / n+2)-1} \eta^{1 / n+2} \theta(\eta) d \eta .
$$

Using the definition of $a, \mathrm{Eq}$. (3.3), gives

$$
\frac{C}{2}=(1 / n+2)^{n / n+2} \frac{\Gamma(1 / n+2)}{\Gamma(2 / n+2) \Gamma(n+1 / n+2)} \int_{0}^{1}(1-\eta)^{(1 / n+2)-1} \eta^{1 / n+2} \theta(\eta) d \eta .
$$

\section{REFERENCES}

1. M. J. Lighthill, Reflection at a laminar boundary layer of a weak steady disturbance to a supersonic stream, neglecting viscosity and heat conduction, Quart. J. Mech. and Appl. Math. III-3, 305-325 (1950)

2. C. C. Chang and J. Werner, A solution of the telegraph equation with application to two dimensional supersonic shear flow, J. Math. and Phys. 31, 91-101 (1952)

3. G. N. Ward, Linearized theory of steady high-speed flow, Cambridge Univ. Press, 1955

4. F. Tricomi, On linear partial differential equations of second order of mixed type, translation A9-T-26 Graduate Division of Applied Mathematics, Brown University, 1948

5. R. Paley and N. Wiener, The Fourier-transform in the complex domain, Am. Math. Soc., Colloquium Publ., 1934

6. P. M. Morse and H. Feshbach, Methods of theoretical physics, McGraw-Hill, New York, 1953

7. G. F. Carrier, A generalization of the Wiener-Hopf technique, Quart. Appl. Math. 7, 105-109 (1949)

8. G. N. Watson, Bessel functions, Cambridge Univ. Press, 1954

9. J. A. Lewis and G. F. Carrier, Some remarks on the flat plate boundary layer, Quart. Appl. Math. 7, 228-234 (1949)

10. A. Erdelyi, W. Magnus, etc., Tables of integral transforms, vols. 1, 2, McGraw-Hill, New York, 1954

11. E. T. Whittaker and G. N. Watson, Modern analysis, Cambridge Univ. Press, 1935 